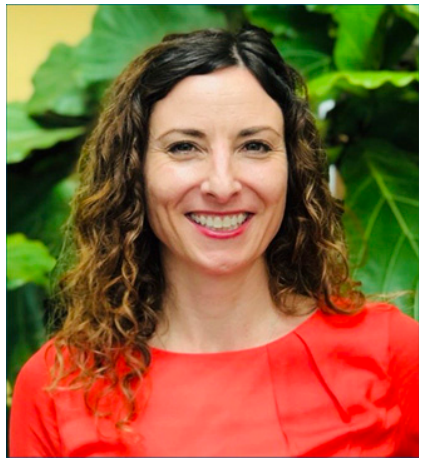

JACQUELINE L. BENDER, PhD

Jacqueline L. Bender, PhD, is a Scientist in the Cancer Rehabilitation and Survivorship Program in the Department of Supportive Care at the Princess Margaret Cancer Centre in Toronto, Canada. She is also an Assistant Professor in the Dalla Lana School of Public Health and the Institute for Health Policy, Management and Evaluation, at the University of Toronto. Dr. Bender's program of research is focused on digital health, cancer survivorship, and implementation science. doi: $10.6004 /$ jnccn.2020.7672

The ideas and viewpoints expressed in this commentary are those of the author and do not necessarily represent any policy, position, or program of NCCN.

\section{The Internet as a Route to Health Information, Support, and Healthcare for Cancer Survivors}

\author{
Jacqueline L. Bender, PhD
}

W ith advances in detection and increasingly sophisticated treatments, twothirds of people who are diagnosed with cancer will become long-term survivors. ${ }^{1}$ This means that most cancer survivors will live $>5$ years after their diagnosis. ${ }^{1}$ In fact, it is estimated that there are $>16.9$ million cancer survivors alive in the United States today, and that number is expected to grow to $\geq 22.2$ million by 2030 with the growth and aging of our population. ${ }^{2}$

The growing number of cancer survivors represents both a success and a challenge, which will require innovative models of care and support. ${ }^{3}$ This includes internet use. ${ }^{4}$ As explained by Young Adult Cancer Canada, "it takes longer to recover from cancer than to treat it" (Karine Chalifour, BSW, Young Adult Cancer Canada, personal email communication, October 2020). After treatment, cancer survivors must cope with multiple physical, functional, and psychosocial challenges that can diminish their ability to participate fully in work and life and reduce their overall quality of life..$^{5-7}$ Many of these supportive care issues are neglected or not adequately addressed because of the fractured nature of care delivery, the lack of survivorship programs or processes or incentives to permit their timely management, $3,8,9$ or the patient's location..$^{10}$ As a result, many patients with cancer report being dissatisfied with the information and support they receive from health professionals, ${ }^{11}$ and struggle with high levels of unmet supportive care needs. ${ }^{12}$

The internet offers a convenient and cost-effective way for cancer survivors to access personally tailored health information and support. ${ }^{13-15}$ Relevant information from credible health websites can dispel fears, enhance feelings of control, promote self-care, and decrease anxiety, ${ }^{13,14}$ and emotional support from online groups can buffer distress by normalizing experiences, thereby lessening negative appraisals of events and promoting coping efforts. ${ }^{15}$

Nationally representative, up-to-date data on the prevalence of internet use is needed to inform patient education and supportive care services for cancer survivors. In this issue of JNCCN, using 2013-2018 data from the National Health Interview Survey, Dee et al ${ }^{4}$ report on a cross-sectional analysis of general and health-related internet use among cancer survivors in the United States. In this study, more than two-thirds of respondents were internet users, with internet usage increasing over the 5-year period studied. Among this sample of 12,970 adult survivors, general internet use increased from $63.2 \%$ to $70.8 \%$, and healthspecific internet use increased from 48.9\% to 52.2\% between 2013 and 2018. Other population-based studies using data from the Health Information National Trends Survey have reported similar internet use rates among cancer survivors in the United States. ${ }^{16}$

Although the internet has the potential to reduce healthcare inequalities by providing individuals with access to information and support that might otherwise be inaccessible, ${ }^{10}$ experts have concerns that it may conversely perpetuate healthcare disparities by keeping certain segments of the population

\section{See page 1468 for related article.}


on the sidelines. The study by Dee et $\mathrm{al}^{4}$ serves as an important reminder that disparities in internet access persist among US cancer survivors. Age, education, income, and broadband access are well-established determinants of the digital divide. ${ }^{17}$ Factors associated with general and healthrelated internet use in Dee et al's adjusted multivariable analyses were younger age, female sex, white race (vs Black/ African-American), higher educational attainment, and higher family income. Other population-based studies of cancer survivors in the United State have reported a similar gap in internet use based on sociodemographic factors. ${ }^{14,16}$ In contrast, in a population-based study of prostate cancer survivors in Canada, age, education, and income did not play a significant role in determining health-related Internet use when other factors were considered. ${ }^{18}$ Instead, being an active information seeker, having higher eHealth confidence, and broadband access were the strongest determinants of health-related Internet use. ${ }^{18}$

Notably, Dee et $\mathrm{al}^{4}$ found that cancer survivors who were very dissatisfied with their healthcare were $78 \%$ more likely to use the Internet as a health information source when compared with those who were satisfied and that this association was greater among women. Previous research has shown that women tend to be less satisfied with their healthcare ${ }^{19,20}$ and more likely to seek health information on the internet than men. ${ }^{21}$ In particular, Elliott et al ${ }^{20}$ found that women who were older, less healthy, more educated, non-Hispanic white, or received care in for-profit hospitals were more likely to have somewhat worse patient experiences than men.

Various factors have been proposed to explain these differences between male and female patients. Greater dissatisfaction with healthcare among women may be because they make greater use of and have more experiences with healthcare, and as a result have higher expectations. ${ }^{19}$ Women are also more likely to engage in active coping strategies and to seek social support, ${ }^{22}$ whereas men tend to be more passive information gatherers ${ }^{23}$ or to avoid or deny the stressor entirely. ${ }^{24}$ Evidence suggests that these differences in the way male and female patients cope likely reflect the different roles they assume in society and the different stressors they face and should be taken into account. ${ }^{22}$

For many, the quality of health information on the internet is an enduring concern, and Dee et $\mathrm{al}^{4}$ rightly emphasized the need to improve the quality of health information websites. Indeed, systematic reviews assessing the quality of health information online have consistently shown that health websites tend to be lacking in currency, attribution, balance of evidence, readability, and comprehensiveness. ${ }^{25-28}$ Although efforts to improve the quality of health information online are certainly needed, the participatory nature of the web means that the internet will continue to include unvetted health information. Therefore, of equal importance are efforts to boost the eHealth literacy of cancer survivors and their ability to triangulate information from multiple sources and participate in improving health information online. eHealth literacy interventions offer one potential solution and have been shown to improve knowledge, skills, and health literacy efficacy among seniors. ${ }^{29}$ Further, studies of online cancer support groups have shown that cancer survivors who feel competent deciphering good from bad information put effort into ensuring that the information they share is accurate, often including original sources. ${ }^{14}$ For example, an analysis of the accuracy of 4,600 postings in a breast cancer online support group found only 10 containing false or misleading information. ${ }^{30}$ Of these, 7 were identified as false or misleading by members of the list and corrected, on average, $<5$ hours after they appeared. ${ }^{30}$

The internet also has the potential to transform where and how healthcare is delivered, allowing more accessible, efficient, cost-effective, and patient-centered healthcare. Despite these potential advantages, however, until recently the adoption of virtual care has been limited due to significant technical, logistic, and regulatory challenges. ${ }^{31}$ However, in response to the COVID-19 pandemic, healthcare systems around the world rapidly transitioned to virtual care to control the spread of the virus and protect public safety. For many cancer survivors, this has meant that in-person follow-up appointments were replaced with web-based video visits. Although this new method of healthcare delivery may be a welcome alternative for many cancer survivors-similar to the findings by Dee et al-a large cohort study on the use of virtual care during the COVID-19 pandemic suggests that virtual care may exacerbate healthcare disparities for others. ${ }^{32}$ Specifically, those who were Black, with a home zip code with a lower mean income, and with higher average household size were less likely to use telemedicine to seek care. ${ }^{32}$ At the same time, the study found an increase in the proportion of Black patients accessing urgent virtual care from 2019 to 2020 , driven by a younger, female population. ${ }^{32}$

In summary, given the increasing number of cancer survivors, the growth in health-related internet use among this population, and the likelihood that both trends will continue, the internet should continue to be leveraged to provide cancer survivors with personally tailored information and support services to manage the late and long-term effects of cancer and its treatment. At the same time, persistent disparities in access to the internet must be considered, and systematic approaches to lowering barriers-such as universal broadband internet access and efforts to increase health literacy, among others - must be prioritized for digital health to achieve its potential of improved access to health for all.

Disclosure: Dr. Bender has disclosed that she has no financial interests, arrangements, or affiliations with the manufacturers of any products discussed in this article or their competitors.

Correspondence: Jacqueline L Bender, PhD, Cancer Rehabilitation and Survivorship, Department of Supportive Care, Princess Margaret Cancer Centre, University Health Network, 585 University Avenue, Toronto, Ontario, Canada M5G 2N2. Email: Jackie.Bender@uhnresearch.ca 


\section{References}

1. Allemani $\mathrm{C}$, Weir HK, Carreira $\mathrm{H}$, et al. Global surveillance of cancer survival 1995-2009: analysis of individual data for 25676887 patients from 279 population-based registries in 67 countries (CONCORD-2). Lancet 2015;385:977-1010.

2. Miller $K D$, Nogueira $L$, Mariotto $A B$, et al. Cancer treatment and survivorship statistics, 2019. CA Cancer J Clin 2019;69:363-385

3. Hewitt M, Greenfield S, Stovall E. From Cancer Patient to Cancer Survivor: Lost In Transition. Washington, DC: National Academies Press; 2006.

4. Dee EC, Muralidhar V, Butler SS, et al. General and health-related internet use among cancer survivors in the United States: a 2013-2018 crosssectional analysis. J Natl Compr Canc Netw 2020;18:1468-1475.

5. Stein KD, Syrjala KL, Andrykowski MA. Physical and psychological longterm and late effects of cancer. Cancer 2008;112:2577-2592.

6. Pergolotti M, Deal A, Reeve B. Activities, function, and health-related quality of life (HRQOL) of older adults with cancer. J Geriatr Oncol 2017;8:249-254.

7. Weaver K, Forsythe L, Reeve B, et al. Mental and physical health-related quality of life among U.S. cancer survivors: population estimates from the 2010 Natinoal Health Interview Survey. Cancer Epidemiol Biomarkers Prev 2012;21:2108-2117.

8. Canadian Cancer Research Alliance. Pan-Canadian Framework for Cancer Survivorship Research. Accessed October 15, 2020. Available at: http:// www.frgs.gouv.qc.ca/documents/11314/6220302/Survivor_Framework_ EN.pdf/12f3dcf4-84f7-4722-8f2d-4afdc2f43aba

9. Silver JK, Baima J, Mayer RS. Impairment-driven cancer rehabilitation: an essential component of quality care and survivorship. CA Cancer J Clin 2013;63:295-317.

10. Khairat $\mathrm{S}$, Haithcoat $\mathrm{T}$, Liu $\mathrm{S}$, et al. Advancing health equity and access using telemedicine: a geospatial assessment. J Am Med Informatics Assoc 2019;26:796-805.

11. Jefford M, Tattersall MHN. Informing and involving cancer patients in their own care. Lancet Oncol 2002;3:629-637.

12. Burg MA, Adorno G, Lopez EDS, et al. Current unmet needs of cancer survivors: analysis of open-ended responses to the American Cancer Society Study of Cancer Survivors II. Cancer 2015;121:623-630.

13. Strecher V. Internet methods for delivering behavioral and health-related interventions (eHealth). Annu Rev Clin Psychol 2007;3:53-76.

14. Hesse BW, Greenberg AJ, Rutten LJF. The role of internet resources in clinical oncology: promises and challenges. Nat Rev Clin Oncol 2016;13: 767-776.

15. Bender J. Cancer virtual communities in the era of personalized medicine: perspectives on scope, use and impact. In: El Morr C, ed. Novel Applications of Virtual Communities in Healthcare Settings. Hershey, PA IGI Global; 2018;56-92.

16. Chou WS, Liu B, Post S, Hesse B. Health-related Internet use among cancer survivors: data from the Health Information National Trends Survey, 2003-2008. J Cancer Surviv 2011;5:263-270.
17. Statistics Canada. Life in the fast lane: how are Canadians managing? Accessed October 15, 2020. Available at: https://www150.statcan.gc.ca/ $\mathrm{n} 1 /$ daily-quotidien/171114/dq171114a-eng.htm

18. Bender JL, Feldman-Stewart D, et al. Health-related internet use among men with prostate cancer in Canada: Cancer Registry Survey Study. J Med Internet Res 2019;21:e14241.

19. Coyle J. Exploring the meaning of 'dissatisfaction' with health care: the importance of 'personal identity threat.' Sociol Health Illn 2008;21:95-123.

20. Elliott MN, Lehrman WG, Beckett MK, et al. Gender differences in patients' perceptions of inpatient care. Health Serv Res 2012;47:1482-1501.

21. Adjei Boakye E, Mohammed KA, Geneus CJ, et al. Correlates of health information seeking between adults diagnosed with and without cancer PLoS One 2018;13:e0196446.

22. Tamres LK, Janicki D, Helgeson VS. Sex differences in coping behavior: a meta-analytic review and an examination of relative coping. Personal Soc Psychol Rev 2002;6:2-30.

23. Saab MM, Reidy M, Hegarty J, et al. Men's information-seeking behavio regarding cancer risk and screening: a meta-narrative systematic review. Psychooncology 2018;27:410-419.

24. Oliffe JL, Rossnagel E, Bottorff JL, et al. Community-based men's health promotion programs: eight lessons learnt and their caveats [published online October 11, 2019]. Health Promot Int, doi: https://doi.org/ 10.1093/heapro/daz101

25. Eysenbach G, Powell J, Kuss O, Sa ER. Empirical studies assessing the quality of health information for consumers on the World Wide Web: a systematic review. JAMA 2002;287:2691-2700.

26. Daraz L, Morrow AS, Ponce OJ, et al. Readability of online health information: a meta-narrative systematic review. Am J Med Qual 2018; 33:487-492.

27. Black PC, Penson DF. Prostate cancer on the internet-information or misinformation? J Urol 2006;175:1836-1842.

28. Kobes K, Harris IB, Regehr G, et al. Malignant websites? Analyzing the quality of prostate cancer education web resources. Can Urol Assoc 2018;12:344-350.

29. Xie B. Effects of an ehealth literacy intervention for older adults. J Med Internet Res 2011;13:e90.

30. Esquivel A, Meric-Bernstam F, Bernstam E V. Accuracy and self correction of information received from an internet breast cancer list: content analysis. BMJ 2006;332:939-942.

31. Canadian Partnership Against Cancer. Virtual care in Canada: an environmental scan. Accessed October 15, 2020. Available at: https://www.partnershipagainstcancer.ca/topics/virtual-care-canada/

32. Chunara R, Zhao Y, Chen J, et al. Telemedicine and healthcare disparities a cohort study in a large healthcare system in New York City during COVID-19 [published onine August 31, 2020]. J Am Med Informatics Assoc, doi: https://doi.org/10.1093/jamia/ocaa217 